\section{Lumbar Disc Nomenclature: Version}

\section{0}

\section{A.L. Williams, F.R. Murtagh, S.L.G. Rothman, and G.K. Sze}

T he above document by Fardon et al, listed in this issue's Table of Contents with links to the full versions recently published by Spine and The Spine Journal, represents an update to the original article coauthored by David Fardon, MD, and Pierre Milette, $\mathrm{MD}$, entitled "Nomenclature and Classification of Lumbar Disk Pathology: Recommendations of the Combined Task Forces of the American Society of Spine Radiology, the American Society of Neuroradiology and the North American Spine Society" and published in Spine in 2001.

Version 2.0 updates the initial article, which provided a standardized nomenclature used extensively by imaging and clinical physicians over the past 13 years. Although the original document has stood the test of time, some sections and definitions were accepted more readily than others. Responding to an initiative from the American Society of Spine Radiology, a task force of spine physicians from the American Society of Spine Radiology, American Society of Neuroradiology, and North American Spine Society has reviewed and modified the original document. This revision represents the end-result of what turned out to be a 10year process. The revision preserves the format and much of the language of the original document. The general principles that guided the original document remain unchanged. Definitions are based on anatomy and pathology, primarily as visualized on imaging studies. Definitions of diagnoses are not intended to imply external etiologic events such as trauma, do not imply relationship to symptoms, and do not define or imply need for specific treatment.

The modifications to the original document deal primarily with 1) updating and expanding the text, glossary, and references; 2) revision of the distinction between disk herniation and asymmetrically bulging disk; 3) revision of the illustrations; 4) emphasis of the term "annular fissure" in place of "annular tear"; 5) refinement of the definitions of "acute" and "chronic" disk her-

http://dx.doi.org/10.3174/ajnr.A4108 niation and other minor amendments. It is hoped that these disk nomenclature modifications will encourage use by all physicians involved in the diagnosis and treatment of spinal diseases in their daily practice.

Why is disk nomenclature important? In an AJNR editorial in January 2007, Reed Murtagh, MD, a senior member of our society, stated that "we need language to communicate precisely and objectively." Galen of Pergamon (129-199 AD), a Roman physician, surgeon, and philosopher, said that "the chief merit of language is clearness, and we know that nothing detracts so much from this as do unfamiliar terms." As neuroradiologists, we communicate with a diverse number of subgroups, including referring physicians, trainees, insurance companies, patients, and attorneys. The key to useful communication with professionals in these groups is the uniformity or standardization of definitions.

The standardization of terms positively impacts our practice of medicine in many ways. With respect to patient care, it is imperative that radiologists and clinicians understand one another. Patient treatment regimens depend on it. Follow-up imaging studies may be read by different radiologists. Different clinicians may be following the same patient. It is important for residents and fellows to learn standardized nomenclature from the outset of their training (eg, Disk Nomenclature 101). Research activities require uniform nomenclature for reliable data collection, indexing, and mining. In the medical-legal realm, attorneys need to understand the content of radiology reports. RadLex, a comprehensive lexicon of radiology terms for the standardized organizing, indexing, and retrieving of radiology information, relies on standardized terms. Structured report templates similar to those used in mammography (eg, BI-RADS) seem to be gaining favor in the "new" medical environment. Disk nomenclature is crucial in such a system.

In conclusion, it is hoped that this revised document will gain even wider acceptance than did the original among all physicians involved in the diagnosis and treatment of spinal diseases. The authors, members of the American Society of Spine Radiology/ American Society of Neuroradiology Nomenclature Committee, encourage our neuroradiology colleagues to review the revised document and incorporate this lumbar disk nomenclature in their daily practice. 